\title{
Economics of Information Processing in Operations Organizations
}

\author{
Phillip J. Lederer
}

\author{
William E. Simon School of Business Administration \\ lederer@simon.rochester.edu
}

\begin{abstract}
This paper studies a fundamental management question: how does information economics affect the organization of management? We view management hierarchies as tree-like structures designed to minimize real and opportunity costs related to information processing and decision making. "Line" production activities stand at the end nodes of a hierarchy tree. Data from these bottom nodes are processed and distributed to higher level nodes that combine information from the lower nodes. The question we ask is: "how do the real and opportunity costs of information processing affect the tree". We solve for the optimal tree which includes the links and capacity at each of the nodes. Models are formulated on two underlying premises: complexity costs arise due to processing different types of data, and queuing effects due to data arrival and processing uncertainties create delay which is an opportunity cost.
\end{abstract}

\section{INTRODUCTION}

Management systems are typically organized as a hierarchy and exists to monitor, coordinate, control and make decisions about productive internal activities that lead to final products. It is often overlooked that these functions are information intensive and the economics of information processing has much to do with the design of management hierarchy. My view is that management hierarchy specifically exists to efficiently process information. The goal of this paper is to better understand how the economics of efficient information processing affects the design of management hierarchy.

Many research papers have generated insights about principles of hierarchy design by focusing on specific structural aspects, e. g. scale economies [1], and organization for information processing [2], etc. However unrealistic assumptions are made in this literature regarding the process of information processing. For example, it is largely assumed that the basic organizational unit is a single individual (thus is not scalable). Nodes in a hierarchy represent "departments" with one manager! In this literature it is most often assumed that the process of capturing and processing data into information is deterministic.
In these models, delay occurs in a cycle time like way: delay is caused by the time it takes for a worker to process the pile of data messages on his/her's desk!

Instead we model management hierarchy by focusing on departmental structure where the capacity of a department is endogenous. Two underlying assumptions provide the foundation of our model. First, that uncertainty causes delay in processing data into information, thus queueing, and second, that processing data becomes more difficult when a manager is asked to process from a variety of sources.

Costs are generated by the expense of staffing departments and the opportunity cost of delay. We assume that the hierarchy design corresponds to the hierarchy with the cost minimizing structure. It is assumed that data originates from the hierarchy's roots called data sources where operations tasks are actually performed by small workgroups and/or equipment. Process scope is the number of data sources that generate operating data that needs to be analyzed. Higher level nodes in the hierarchy correspond to departments staffed by managers who process data from lower level departments, possibly make decisions and then pass processed information to the next higher level. A department is staffed by multiple individuals and computing resources needed to process incoming data and information. Typically there are multiple departments at each tier, but at the top, only one.

The capacity in each department is measured by its processing rate, which is a function of the number of staff and/or amount of equipment devoted to information processing. These resources are costly. Also, delays in processing data causes opportunity cost. Short response times add value by helping a firm make timely error-free decisions and respond to coordination problems, while adjusting to changing market conditions quicker and serving customers faster. (See [3] for further elaboration of the value of timely service.)

Queuing is a basic universal, physical process in any productive system in which uncertainty in arrival patterns or processing times exists, and is understood to have significant economies of scale. At data centers data is constantly produced, much of it not informative, but issues arise requiring higher 
level interpretation and decision guidance. The incidence of these occasions creates a flow of data from the data sources upward into the hierarchy where it needs to be processed. Randomness of data arrival rates and processing times are more the rule than the exception, introducing queuing which enjoys economies of scale. Economies of scale encourage efficiency by merging data flows and consolidating information processing. In short, it is efficient to reduce the number of departments per tier and increase the number of data streams into above departments. The effect is that the hierarchy becomes flatter.

Without some effect offsetting the queuing economy of scale, the optimal hierarchy will consist of a single processor. This is because with flow aggregation, one can minimize the processing capacity required (subject to a service time constraint) with a single very large processor serving a very high incoming data rate. But it is more realistic to assume complexity related costs: a superadditive cost structure with respect to scope to processing data from a variety of sources. Simply stated, when the number of direct reports to a department rises, management difficulty rises because it takes effort to switch from task to task. Increased management difficulty causes delays in processing data. That might be ameliorated by more processing capacity. But that is the tradeoff we study: the cost of delay versus the cost of capacity.

Because it is generally efficient to limit the number of data streams into departments, higher management levels are needed to fully internalize the effect of all production units on the other production units. That is, to glean information and makes decisions that affects many data sources. Thus the hierarchy gets taller.

\subsection{Summary of results}

Our analysis predicts how information processing influences the hierarchy's shape. Among the results are that large increases in information processing cost/unit and large increases in data flow rates don't change the hierarchy's departmental structure very much but greatly affect capacity allocation decisions for departments. Process scope diseconomies exist when data sources are few, but this changes to scope economies with many sources. The implication is that decomposition of the hierarchy into smaller sets of data sources is inefficient.

We show that the number of subordinates of a department increases as one rises in the hierarchy. Increasing span means higher level managers do less specialized work which seems intuitively reasonable. In many papers the opposite is claimed: see [4] and [5]. Also, as one moves upward in the hierarchy, the capacity allocated to each department rises while the aggregate capacity at each tier falls, which is in agreement with many existing papers.

Due to queuing, utilization and delay falls as one ascends tiers. This means that the effective workload of higher management is less than at lower tiers. We find that higher level departments have more subordinates.

General patterns emerge for changes in department structure with changes in structural parameters. With increased scale (that is, data flow rates) or process scope (number of data) the number of tiers in the hierarchy rise, but at a decreasing rate. Capacity cost per unit output has only a second order effect on hierarchy height. A similar statement can be said of data flow. Analysis and examples show that increasing the delay cost rate per unit time (or reducing the cost of processing capacity) causes the hierarchy to shrink. Intuitively, higher delay cost reduces the relative importance of capacity cost which causes more capacity to be employed and the number of subordinates to rise for all departments. This increase in subordinates implies that there are more departments at remaining tiers, but the increase can be small. In an example we show that even with a 4-fold increase in the importance of delay, the total capacity employed rises by a small amount (for example a $10 \%$ increase) and the number of departments at remaining tiers is only slightly greater than before.

Many factors cause more specialization. Specialization refers to processing a narrower range of data types coming from data sources. A department's specialization increases with higher data flow rates, higher complexity cost, higher delay cost, and larger process scope. However, unless one of these factors changes a great deal, the general departmental structure (and thus specialization) does not change much. Instead changes in these factors cause the allocation of capacity to shift greatly.

\section{MODELS OF HIERARCHY}

A hierarchy is a topological tree where all nodes except those at the bottom correspond to informationprocessing departments, and the bottom nodes are data sources. Nodes correspond to department which is where data processing occurs. Departments pass information upward to departments higher up. This process of receiving and processing data, and then passing it upwards in the hierarchy continues until 
there is just one department at the top of the hierarchy. Figure 1 illustrates the hierarchy.

The shape of a hierarchy can be described by the number of tiers or levels of management, each department's span of control and each department's processing capacity. If the span of control is large for each department then the hierarchy is flatter and decisions are made faster but at the cost of extra capacity due to added complexity. In contrast, a hierarchy that is "taller" exhibits the opposite advantages and disadvantages. Hence, the costs incurred in an information-processing hierarchy are highly associated with its shape.

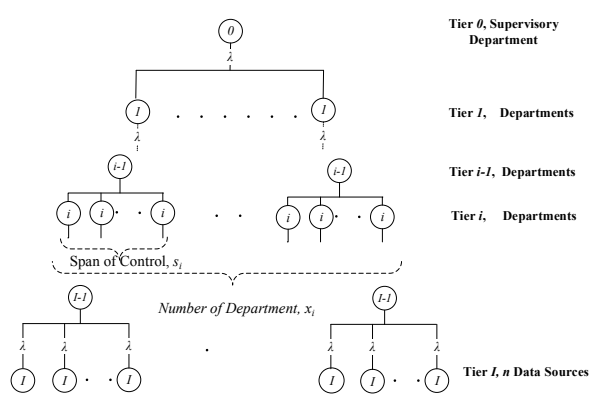

FIGURE 1: A Hierarchy

\subsection{Model setup}

We assume a symmetric hierarchy as shown in Figure 1. Data is generated by random events according to a Poisson process with rate $\lambda$ where larger $\lambda$ means higher data flow rates need to be processed. The time delay is the total time for the data to traverse from the bottom to the top of the hierarchy. The number of departments (or data sources at the bottom level) of each tier $i$ is denoted by $x_{i}$ and the span of control of each department at tier $i$ is denoted by $s_{i}$. Each department at the $i$ th tier receives $\lambda * s_{i+1}$ data signals per unit time from its subordinates Time to process data is assumed to follow an exponential distribution. At each department, time delay is modeled as an $\mathrm{M} / \mathrm{M} / 1$ queue. Rate $\mu$ is the total data processing rate at tier $i$ and is our measure of capacity. Thus, the capacity per department is $\mu / x$. The effective processing rate of each department is reduced by the complexity of arriving work. Complexity is set by the number of subordinates a department serves, say, $s$ and also a parameter $\delta$ : $0 \leq \delta \leq 2$. $A$ department's effective processing rate is $\mu / x S^{\delta}$. If at tier $i$, there are $x_{i}$ departments each with $s_{i}$ subordinates and the capacity has been set to $\mu_{i}$, then, the time delay at each department at tier $i$ is just

$$
l_{i}=\frac{1}{\frac{\mu_{i}}{s_{i} \delta} \frac{1}{x}-\lambda} .
$$

Thus, the otal delay time to process data from source to apex is just $L=\sum_{i=0}^{I-1} l_{i}$

We denote the cost rate of capacity by $C_{c}$, and the cost of delay per unit time by $C_{w}$. Table 1 lists the notation we use in this paper.

Table 1: Table of Notation

\begin{tabular}{|c|c|c|}
\hline \multirow[t]{4}{*}{$\begin{array}{l}\text { Exogenous } \\
\text { variables: }\end{array}$} & $\lambda:$ & $\begin{array}{l}\text { Data flow rate/production center and the } \\
\text { firm's production rate, units/time }\end{array}$ \\
\hline & $C_{w}:$ & $\begin{array}{l}\text { Cost of time delay per unit-time, } \$ / \text { unit- } \\
\text { time }\end{array}$ \\
\hline & $C_{c}:$ & $\begin{array}{l}\text { Cost of capacity per unit-time at tier } \\
i, i=1,2, \ldots, I-1, \$ / \text { unit-time }\end{array}$ \\
\hline & $\delta:$ & Complexity level, $1 \leq \delta \leq 2$ \\
\hline \multirow[t]{4}{*}{$\begin{array}{l}\text { Decision } \\
\text { variables: }\end{array}$} & $x_{i}, x(t)$ & $\begin{array}{l}\text { Number of departments at tier } \\
i \text { in integer formulation, at tier } t \text { in contins }\end{array}$ \\
\hline & $s_{i}, s(t):$ & $\begin{array}{l}\text { Span of control at tier } i \text { in integer } \\
\text { formulation, at tier } t \text { in continuous } \\
\text { formulation }\end{array}$ \\
\hline & $\mu_{i}:$ & $\begin{array}{l}\text { Total processing capacity at tier } i, i= \\
1,2, \ldots, I-1 \text {, units/time }\end{array}$ \\
\hline & $I, T:$ & $\begin{array}{l}\text { Number of tiers in integer formulation, in } \\
\text { continuous formulation }\end{array}$ \\
\hline \multirow{2}{*}{$\begin{array}{l}\text { Other } \\
\text { variables: }\end{array}$} & Nor $n:$ & Number of data sources \\
\hline & $i, t:$ & $\begin{array}{l}\text { Index of tier in integer, continuous } \\
\text { formulations }\end{array}$ \\
\hline
\end{tabular}

Total cost is the weighted sum of capacity cost and delay cost over the hierachy's $I$ tiers:

$$
T C=\lambda C_{w} L+\sum_{i=0}^{I-1} C_{c} \mu_{i}
$$

The number of departments in each tier $i$ can be expressed as $x_{i}=s_{0} s_{1} s_{2} \ldots s_{i-1}$. Given equations and (1), the total cost when there are $I$ tiers in the hierarchy is

$$
T C=\sum_{i=0}^{I-1}\left(\frac{\lambda C_{w}}{\frac{\mu_{i}}{x_{i}} \frac{1}{\left(x_{i+1} / x_{i}\right)^{\delta}}-\lambda}+C_{c} \mu_{i}\right)
$$

The total cost function is concave in $\mu_{i}, \forall i$. Minimizing the total cost with respect to $\mu_{i}$ gives the cost minimizing $\mu_{i}$ as

$$
\mu_{i}=\sqrt{\frac{\lambda C_{w} x_{i}\left(\frac{x_{i+1}}{x_{i}}\right)^{\delta}}{C_{c}}}+x_{i}\left(\frac{x_{i+1}}{x_{i}}\right)^{\delta} \lambda .
$$

As is shown, capacity choice is a variable that is set by the number of departments. Thus, this equation by itself cannot deliver insightful insights.

If one substitutes the expression for optimal $\mu_{i}$ back into the total cost function it appears that total cost is expressed as 
$T C=\sum_{i=0}^{I-1} f\left(x_{i}, x_{i+1}, C_{c}, C_{w}, \lambda, \delta\right)$ where

$f\left(x_{i}, x_{i+1}, C_{c}, C_{w}, \lambda, \delta\right)=2 \sqrt{\lambda C_{c} C_{w} x_{i}\left(\frac{x_{i+1}}{x_{i}}\right)^{\delta}}+\lambda C_{c} x_{i}\left(\frac{x_{i+1}}{x_{i}}\right)^{\delta}$

as the cost of capacity and time delay at tier $i$. Note that $x_{0}=1$ and $x_{I}=n$. Additional integer constraints are imposed on the decision variables. The resulting integer optimization problem can be expressed as follows:

$$
\min _{x_{i}, I} \sum_{i=0}^{I-1} f\left(x_{i}, x_{i+1}, C_{c}, C_{w}, \lambda, \delta\right)
$$

subject

$$
\begin{gathered}
s_{i}=\frac{x_{i+1}}{x_{i}} \\
i=0,1, \ldots, I-1, \\
s_{i}, \text { and } x_{i} \text { are integers, }
\end{gathered}
$$

and

$$
x_{0}=1, x_{I}=n
$$

This is an open-ended calculus of variations problem with $x_{i}$ being the state variable and $s_{i}$ the control variable.

Optimal hierarchies can be found by direct computation of all the possible hierarchy designs. Although in principle this sort of computation can be done, this approach does not lead to general analytical insights. Instead, to do so we apply a continuous-tier approximation to the hierarchy design problem.

\subsection{Continuous-tier approximation}

In this section, we drop the integer constraints and solve the hierarchy design problem by using a continuous-tier approximation. We use the positive real number $t$ instead of integer $i$ to represent the number of tiers in the hierarchy, $x_{i}$ and $s_{i}$ are replaced by $x(t)$ and $s(t)$ and $I$ become $T$. The number of departments at tier $I$ in the integer representation is just $x_{i}=s_{0} s_{1} s_{2} \ldots s_{i-1}$ and in can be can be rewritten in the form $\ln \left(x_{i}\right)=\sum_{j=0}^{i} \ln \left(s_{j}\right)$. Accordingly, in the continuous representation it becomes $\ln x(t)=\int_{0}^{t} \ln s(u) d u$. Differentiating both sides with respect to $t$ gives $x^{\prime}(t)=$ $x(t) \ln (s(t)) . \quad$ We replace $\left(x_{i+1} / x_{i}\right)^{\delta} \quad$ with $\ln \left(s(t)^{\delta}\right)$ and the rest of $x_{i}$ with $x(t)$ implying $(s(t))^{\delta}=e^{\frac{\delta x^{\prime}(t)}{x(t)}}$. Thus, the continuous form of our problem is as follows:

$$
\min _{T, x(t)} \int_{t=0}^{T} f\left(x(t) x^{\prime}(t) d t,\right.
$$

Subject

$$
x(0)=1, x(T)=n \text {, }
$$

Where $\quad f\left(x(t), x^{\prime}(t)\right)=$

$$
2 \sqrt{\lambda C_{c} C_{w} x(t) e^{\frac{x^{\prime}(t) \delta}{x(t)}}}+\lambda C_{c} x(t) e^{\frac{x^{\prime}(t) \delta}{x(t)}},
$$

And $x(t)$ and $T$ are a decision variables.

Referring to section 9 in [6], our problem belongs to the category of calculus of variations problems with free horizons, that is, $T$ is free and $x(T)$ is fixed at the value of $n$. The optimal solution satisfies the following conditions:

$$
\begin{gathered}
\frac{\partial f}{\partial x}-\frac{d}{d t}\left(\frac{\partial f}{\partial x^{\prime}}\right)=0, \\
f-x^{\prime}(t) \frac{\partial f}{\partial x^{\prime}}=0, \text { at } t=T, \\
x(T)-n=0, \\
x(0)=1 .
\end{gathered}
$$

and

This problem satisfies sufficient conditions for a solution to exist. Equations (5.1) and (5.2) are the Euler equation and the transversality condition, respectively. Substituting (4.1) in equations (5.1) and (5.2) gives two equations in $x(t), x^{\prime}(t), x^{\prime \prime}(t)$, and $x^{\prime}(T)$. Accompanied by equations (5.3) and (5.4), numerical methods can find $x(t)$. However, analytic results can be obtained from the problem formulation as we show next.

\subsection{Analytical Results and Illustrations}

Theoretically speaking, solving the equation system of (5.1) to (5.4) will give the solutions to $T$ and $x(t)$, for the optimal hierarchical structure. We start with studying the Euler equation. Substituting (4.3) in equation (5.1) gives

$$
\begin{array}{r}
{\left[\sqrt{\frac{\lambda C_{c} C_{w}}{x(t)} e^{\frac{x^{\prime}(t) \delta}{x(t)}}}\left(2-\delta \frac{x^{\prime}(t)}{x(t)}-\delta^{2} \frac{x^{\prime \prime}(t) x(t)-x^{\prime}(t)^{2}}{x(t)^{2}}\right)\right]} \\
+\left[2 \lambda C_{c} e^{\frac{x^{\prime}(t) \delta(t)}{x(t)}}\left(\begin{array}{c}
1-\delta \frac{x^{\prime}(t)}{x(t)}- \\
\delta^{2} \frac{x^{\prime \prime}(t) x(t)-x^{\prime}(t)^{2}}{x(t)^{2}}
\end{array}\right)\right]
\end{array}
$$

Moreover, substituting (4.3) in the transversality equation (5.2) gives the boundary condition

$$
2 \sqrt{\lambda C_{c} C_{w} n e^{\frac{x^{\prime}(T) \delta}{n}}}+\lambda C_{c} n e^{\frac{x^{\prime}(T) \delta}{n}}
$$




$$
-\left(\sqrt{\lambda C_{c} C_{w} n e^{\frac{x^{\prime}(T) \delta}{n}}}+\lambda C_{c} n e^{\frac{x^{\prime}(T) \delta}{n}}\right) \frac{x^{\prime}(T) \delta}{n}=0
$$

which can be simplified to:

$$
\begin{aligned}
& {\left[\sqrt{\lambda C_{c} C_{w} n e^{\frac{x^{\prime}(T) \delta}{n}}}\left(2-\frac{x^{\prime}(T) \delta}{n}\right)\right]} \\
& +\left[C_{c} n e^{\frac{x^{\prime}(T) \delta}{n}}\left(1-\frac{x^{\prime}(T) \delta}{n}\right)\right]=0 .
\end{aligned}
$$

For fixed $n$, we seek the optimal function $x(t)$ and tier height $T$ but these equations have no closed form solution. But this system can be solved numerically.

The following propositions describe the hierarchy and are presented without proofs which are found in [7].

Proposition 1.: The number of departments at each tier convexly increases from the top to the bottom of the hierarchy, $x^{\prime}(t)>0$, and $x^{\prime \prime}(t)>0$.

Proposition 2.: The span of control falls from the top to the bottom of the hierarchy: $s^{\prime}(t)<0$.

Proposition 1 suggests the "trumpet-like" department structure as seen in Figure 2. Intuitively, the number of departments falls as one ascends. Insight can be gained by considering that a unit of capacity reduces delay much more at the top of the hierarchy than at any lower tier. At each tier, any added capacity must be divided up among the departments, thus there is less "division" higher up and each department will get more. Thus, it is rational to allocate proportionally more capacity to departments higher up, which in turn allows the departments to efficiently process data from more subordinates.

Thus the number of subordinates in the hierarchy rises as one ascends.

As to the capacity in an optimal hierarchy, the following proposition generalizes how capacity is allocated at each tier.

Proposition 3.: The capacity per department rises and the total capacity at a tier falls from the bottom to the top of the hierarchy.

A higher level department is endowed with more capacity because it has to coordinate more subordinates. Increased complexity reduces information-processing efficiency, thus requiring more capacity.

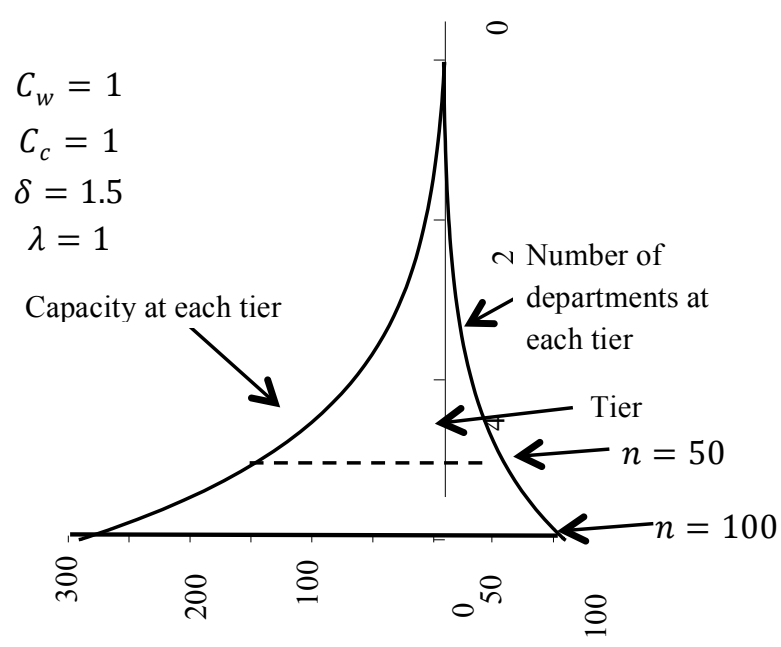

FIGURE 2: The graph demonstrates that when the number of data sources differ, common tiers from the top possess identical department sizes and capacities.

It turns out that capacity is so effective at reducing delay at higher tiers that the utilization of departments (and its workers) actually falls as one ascends the hierarchy.

Proposition 4.: The utilization of each department falls as one ascends the hierarchy.

Given that the capacity cost at each tier is the same and is a proxy for wages, the result states that managers at higher levels actually work less intensively than lower level supervisors. Intuitively, utilization falls because reducing delay at the top is less costly than doing the same at lower levels. In a queuing system, reducing delay is equivalent to lowering capacity utilization). On the contrary, many papers in the literature assume high tier managers earn a higher wage rate than lower tier managers, and this effect increases the utilization as one rises. This does not occur in our model: at higher tiers, the increasing number of subordinates convexly raises the effective cost of processing data. It is clear that the current pattern will be reversed if the wage rates rise steeply enough with tier. But, with a steep wage profile by tier, the subordinate count and the delay will be lower than if queuing phenomenon is ignored.

Next, we study how the shape of the optimal hierarchy changes with increased process scope. 
Proposition 5.: When process scope (n) increases, then the optimal number of tiers of a hierarchy concavely increases and the total capacity, total time delay, and the total cost of capacity and delay increase. The average cost first increases and then decreases with the process scope. Further, the existing optimal hierarchy of an organization retains its shape and becomes the top portion of the new hierarchy when the process scope increases.

This positive relationship between the number of tiers and the process scope is not surprising and has been shown empirically and theoretically. The number of departments at each tier and the capacity allocated to each tier fall from the top to the bottom tier. An example of these properties is illustrated in Figure 2 where the y-axis represents the tier number, the left $\mathrm{x}$-axis reports the total capacity by tier, and the right $\mathrm{x}$-axis reports the number of departments by tier. In addition, the figure shows that the optimal hierarchical structure retains its relative shape even when the scope increases. In Figure 2, if the scope is reduced to $n=50$, the shape of this smaller hierarchy is identical to the top portion of the original larger hierarchy above the dashed horizontal line.

The number of departments and the span of control at each tier of a smaller hierarchy are the same as those at the corresponding tiers in a larger hierarchy.

Figure 3 presents analysis of average cost against process scope $(n)$. The average cost illustrated by the upper curve in the figure indicates that the cost exhibits diseconomies of scope when the process variety is very small and economies of scope at high process scope. We note that both average capacity cost and average delay cost have this property. The average delay cost and the average capacity cost have been drawn with a fixed number of subordinates. The graph shows the adjustment in average total cost when an optimal subordinate structure is applied.

Given the assumptions of this section, it can be concluded that if a firm is operating at high process scope levels, from an information economics viewpoint it is inefficient to divide the organization into subunits that are focused on subsets of processes, or in other words, becoming process focused. Such subdivision is paradoxically possible at low scope.

One more observation can be gleaned from this figure: as process scope increases, total delay cost becomes less and less important in hierarchy design. This intuitively follows since delay cost is not weighted by the magnitude of scope. When the product mix is constant, as the number of production centers increases, time delay becomes only a small portion of the hierarchy control cost and becomes a minor factor in hierarchy design when process scope is large. It is this factor which caused past papers to find that the average cost declines with process scope. That holds true here but we also consider economies of scale of queueing.

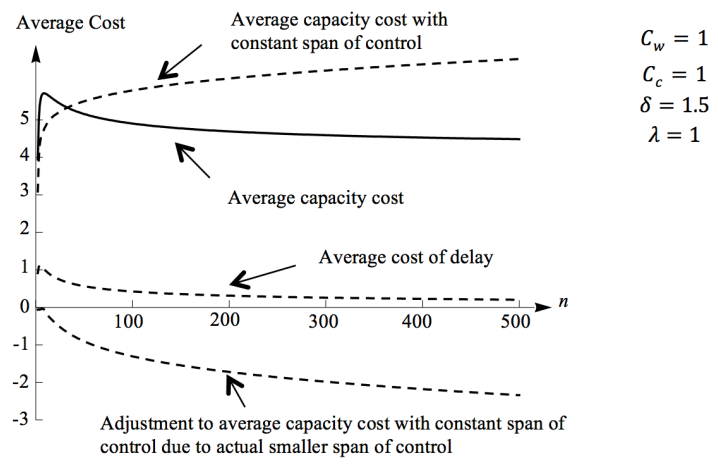

FIGURE 3: The graph shows that for a small number of data sources $(n)$, the average cost rises (diseconomies of scope), especially for capacity cost, but for large process scopes, economies prevail as the average cost declines. The graph also captures the change in average cost due to changes in the optimal subordinate structure.

The structure of a hierarchy is also shaped by data flow scale $(\lambda)$. When scale changes, an organization restructures its hierarchy. The following proposition characterizes the impact of scale.

Proposition 6.: With an increase in the scale ( $\lambda)$, the optimal number of tiers concavely increases and the total capacity, total delay, and total cost increase. Capacity at the top tiers strictly increases while the span of control falls and because tier capacity increases, utilization falls. Because the span of control falls, there are fewer departments at each existing tier. Average cost falls due to economies of scale of queuing and reduced complexity at incremental tiers thus scope economies exist.

Higher scale implies that at corresponding tiers there will be fewer subordinate departments resulting in more specialization and more tiers.

Figure 4 shows that with a ten-fold increase in scale, the number of tiers increases only slightlyFigure 4 shows that with increased scale there is a small shift to specialization. Figure 5 shows that the number of department in a tier gets smaller with 
larger scale and that the hierarchy height only increases a small amount.

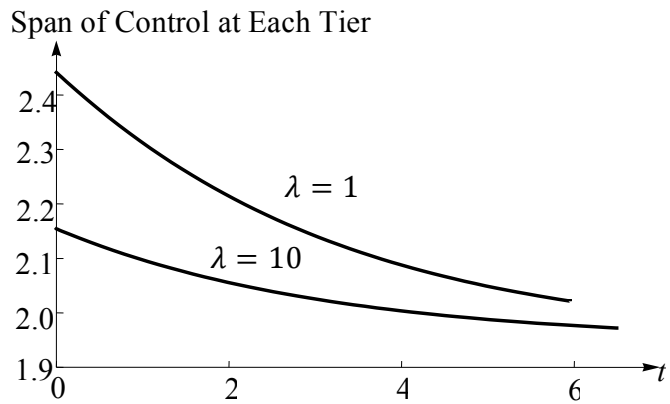

FIGURE 4: Impact of the data flow rate on the span of control. Specialization of departments does increase, but note how little the number of subordinates fall with a tenfold volume increase. This indicates that there are limits to specialization with scale increases.

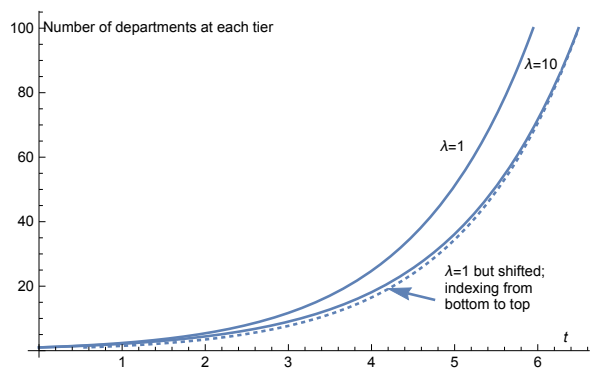

FIGURE 5: Impact of scale on number of departments. This graph demonstrates that the general shape of the hierarchy in terms of number of departments does not change radically with the volume of data. At each tier the number of departments for the $\lambda=10$ case falls compared to the $\lambda=1$ case.

That large shifts in the flow rate cause small changes in hierarchy height will be an effect later observed with the cost parameters, $C_{C}, C_{w}$.

Table 2 reports data comparing performance when $\lambda=1$ and when $\lambda=10$. In this example $n=100$ and when the data flow increases from $\lambda=1$ to $\lambda=10$ total capacity and total cost increase by a factor of 8.8 demonstrating economies of scale.

The complexity of information-processing and coordination is captured by the parameter $\delta$ and we explore how the magnitude of this parameter affects the optimal hierarchical structure, which is characterized by the following proposition.
Proposition 7.: When complexity level $(\delta)$ increases, the optimal number of tiers increases and the total capacity, the total delay, and the total cost of capacity and delay increase

\begin{tabular}{|l|l|c|l|l|}
\hline $\begin{array}{l}\text { Except as } \\
\text { noted } \\
\mathrm{Cc}=1, \\
\mathrm{Cw}=1, \\
\mathrm{n}=100 \\
\lambda=1, \\
\delta=1 .\end{array}$ & $\begin{array}{l}\text { Delay } \\
\text { (time) }\end{array}$ & $\begin{array}{l}\text { Capacity } \\
\text { (units) }\end{array}$ & $\begin{array}{l}\text { Cost } \\
(\$)\end{array}$ \\
\hline $\begin{array}{l}\text { Base case } \\
\text { High delay } \\
\text { cost }\end{array}$ & $\mathrm{Cw}=4$ & $\begin{array}{c}20.8 \\
(49 \%)\end{array}$ & $\begin{array}{c}500.3 \\
(110 \%)\end{array}$ & $\begin{array}{c}573.4 \\
(117 \%)\end{array}$ \\
\hline $\begin{array}{l}\text { Increase } \\
\text { in data } \\
\text { flow }\end{array}$ & $\lambda=10$ & $\begin{array}{c}13.8 \\
(32 \%)\end{array}$ & $\begin{array}{c}4177.0 \\
(920 \%)\end{array}$ & $\begin{array}{l}4315 . \\
(880 \%)\end{array}$ \\
\hline
\end{tabular}

TABLE 2: The delay time, capacity and cost for optimal hierarchies under different structural assumptions. The percentage is a column computation: the fraction of the "base case" number in that column.

In order to counter the effect of increased complexity cost the organization shrinks the span of control and adds capacity to the resulting departments. Increased complexity level, $\delta$, leads to a taller and narrower hierarchy with more specialization at all tiers. Interestingly, total cost is approximately linear in parameter $\delta$.

The optimal hierarchy is also shaped by the relative importance of the two types of costs, the delay cost and the capacity cost. The following proposition illustrates how the number of tiers changes with the ratio of these two costs, $C_{w} / C_{C}$.

Proposition 8.: The optimal number of tiers increases with the ratio, $C_{c} / C_{w}$.

The proposition implies that as the cost of delay (the cost of capacity) becomes larger (smaller), the optimal hierarchy becomes flatter and the number of departments at each level falls. Spans of control increase, reducing specialization and hierarchy height. Higher delay costs cause capacity cost to in effect become relatively smaller causing an increase in capacity in the remaining tiers. Figure 6 shows the distribution of capacity and Figure 7 compares the 


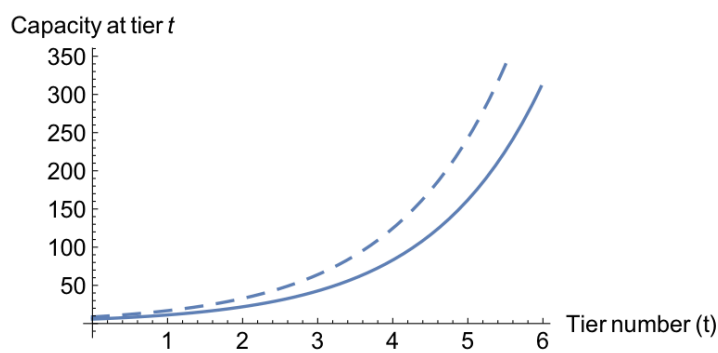

FIGURE 6: Distribution of capacity when $C_{w}=1$ (solid) and when $C_{w}=4$ (dashed). The capacity in each tier rises significantly when the delay cost rate is multiplied by 4.

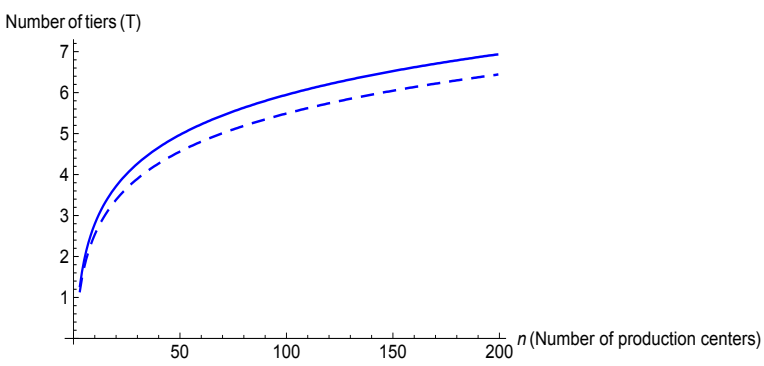

FIGURE 7: The number of tiers vs the number of data sources when $C_{w}=1$ (solid) and when $C_{w}=4$ (dashed). In this example the number of tiers with $n$ data sources does not fall much when the delay cost rate is multiplied by 4 .

tier structure for $n$ data sources when $C_{w}=1$, and when $C_{w}=4$. As expected the number of tiers falls for all values of $n$ but this change is small. Table 2 reports that the net effect of a quadrupling of delay cost is that delay drops by approximately half with an increase in total capacity of $10 \%$, and an increase in total cost of $17 \%$. Capacity resources formerly at the lower tiers are shifted upward to the remaining ones, and $10 \%$ additional capacity is added. Decreased capacity cost makes unit delay cost relatively more important, and results in effects like those described above. As in the discussion about scale, the departmental structure changes little but capacity is shifted significantly.

\section{MANAGERIAL INSIGHTS}

This paper contributes to the literature in information systems in analyzing the implications of information processing on how a firm is organized.

1. Large increases in the cost of delaying data processing do not affect the departmental structure very much. Many have ascribed the flattening of firm's hierarchy to the development of cheaper information processing. Here we show that is not the case. We have shown that re-optimizing the hierarchy with lower capacity cost or higher delay cost rates cause only a small reduction in height. Instead we posit that other management changes better explain this pattern. For example, introducing decentralized decision making can be shown to cause dramatic reduction in hierarchy height.

2. As the number of data sources increases, additional tiers are added to the hierarchy with little change in the number of departments in the existing tiers. The implications follow: consider a firm that merges with another or otherwise increases the scope of its activities. Our model indicates that the top of the new organizational structure will tend to follow the existing hierarchy's departmental and staffing structure. New layers are added to the bottom of the hierarchy where the bulk of the new staff will reside. Management layers will be added above the direct supervisors of production but below the bulk of existing departments. On the other hand, outsourcing or introducing integrated production equipment that allows consolidation of data sources has the opposite effect. We predict the bottom tiers of management will be pared. This is a striking insight into the disappearance of middle management

3. We found that large increases in data volume cause a relatively small increase in hierarchy height and even a small decrease in the number of departments at each existing tier. The major change is a significant rise in staffing levels at each department. Interestingly, our model predicts that capacity utilization will drop at all existing tiers. That is, managers will be less busy in order to make faster decisions.

4. A department at a higher tier than another has more capacity and operates at lower utilization. The utilization is lower in order to speed up control activities. As a result, managers at higher tiers make faster decisions than lower ones and have more resources available to facilitate decision-making. Higher level managers also have more subordinates, thus their work is less specialized than those below. The supervisors at the lowest level have the fewest subordinates. This seems reasonable by the criterion of common sense. However some may argue that this prediction only holds if one ignores the very high wages earned by the very highest executive officers. It is true that a very steep convex increasing wage rate as one ascends can reverse the prediction. We would argue that the top of an executive organization might follow that pattern, but in operating organizations wage profiles are not as steep. 
5. Many factors cause more specialization of control in departments, that is, fewer subordinates: higher data flow rates, more complexity in processing varied data sources, higher capacity cost, and less emphasis on delay.

6. Although delay cost is an opportunity cost, capacity cost is captured by a firm's managerial accounting system, and is manifest in the organization's overhead cost for control. Conjectures about shifts in control overhead are suggested from the examples found in Table 2. Economies of scale of capacity cost are demonstrated throughout, thus the control associated overhead rate will decline with scale. A large increase in the delay cost rate raises expenditures for "management capacity costs", but our examples show that these costs if measured as overhead may rise only moderately.

\section{References}

[1] KEREN, M. and LEVHARI, D. (1983), "The Internal Organization of The Firm and The Shape of Average Costs", The Bell Journal of Economics, 14, 474-486.

[2] RADNER, R., 1993. The organization of decentralized information-processing. Econometrica 61, 1109-1146

[3] LEDERER, P.J. and LI, L. (1997), "Pricing, Production, Scheduling, and Delivery-Time Competition", Operations Research, 45, 407-420.

[4] KEREN, M. and LEVHARI, D. (1979), ."The Optimum Span of Control in a Pure Hierarchy", Management Science, 25, 1162-1172.

[5] BOLTON, P and DEWATRIPONT, M. (1994), "The Firm as a Communication Network", The Quarterly Journal of Economics, 109, 809-839.

[6] KAMIEN, M.I. and SCHWARTZ, N.L. (1991), "Dynamic Optimization: The Calculus of Variations and Optimal Control in Economics and Management", (North-Holland).

[7] LEDERER,P and X. Zheng, (2018), "Information Processing in Management Hierarchy: Scale, Scope and Focus,", Simon School Working Paper. 